\section{A PCT-wide collaborative clinical audit selecting recall intervals for patients according to risk}

\author{
P. J. Cannell ${ }^{1}$
}

VERIFIABLE CPD PAPER
IN BRIEF
- Shows readers how clinical audit can be used as a tool to explore the relationship between recall interval and risk in general dental practice.
- Describes a large collaborative audit design that can be used by many participants.
- Shows behaviour change in relation to recall intervals and risk among participating GDPs.

\begin{abstract}
Aims and objectives This audit was carried out to assess the level to which recall intervals were individually and appropriately selected for patients attending dental practices across a primary care trust (PCT) area in Essex. Method A retrospective audit was carried out by reference to patient records to assess various criteria, including whether patients were categorised according to risk of oral disease, whether an appropriate recall had been selected and whether a discussion regarding a recall interval had been undertaken. An educational event highlighting the issue of recall intervals was held. Subsequent to this a prospective audit was undertaken to assess relevant criteria. Results Prospective audit data showed a marked increase in the use of patient risk assessments for caries, periodontal disease, oral cancer and non-carious tooth surface loss (NCTSL). Recall intervals were also more often selected based on a patient's risk status and discussed with the patient compared to that observed in the retrospective audit data. Conclusion This audit was successful as a tool to bring about change in the behaviour of dentists regarding their determination of appropriate recall intervals for patients. Whether that change in behaviour is long-term or transient requires further investigation.
\end{abstract}

\section{INTRODUCTION}

In September 1995 a voluntary pilot scheme for clinical audit in general dental practice was launched. ${ }^{1}$ The guidance accompanying this scheme proposed the following definition of clinical audit:

'Clinical audit is the systematic, critical analysis of the quality of dental care, including the procedures used for diagnosis and treatment, the use of resources and the resulting outcome and quality of life for the patient.'

The audit process has been described as an 'audit cycle', having the following six stages:"

1. Choosing a subject

2. Deciding what ought to be happening - defining quality

3. Observing what is actually happening

"Senior Lecturer and Programme Lead for Oral Health Science, School of Health and Human Sciences,

University of Essex, Elmer Approach, Southend on Sea, SS1 1LW

${ }^{*}$ Correspondence to: Dr Phillip Cannell

Email: pjcann@essex.ac.uk

Tel: +44 (0)1702 328360

Online article number E8

Refereed Paper - accepted 24 August 2010

DOI: 10.1038/sj.bdj.2011.202

${ }^{\circ}$ British Dental Journal 2011; 210: E8 and comparing with what should be happening

4. Identifying strengths and weaknesses and making changes to the way you work

5. Observing again and seeing if things are getting better

6. Looking at your quality definition and seeing if it needs to be modified in the light of what the first audit showed.

In March 2001 the Department of Health announced a development of this scheme, that from 1 April 2001 all GDPs working in the general dental services (GDS) would be required to participate in a rolling programme of at least 15 hours of clinical audit or peer review activity every three years. ${ }^{3}$

A new dental contract was launched on 1 April 2006. With it has come the devolvement of responsibility for clinical audit to primary care trusts (PCTs) within their clinical governance arrangements. ${ }^{4}$ In this period of significant change in the GDS, the position and role of dental clinical audit appears unclear. In particular, the potential for development of clinical audit and the momentum achieved thus far may be diluted by this lack of clarity and change of working arrangements. ${ }^{5}$
On 6 August 2002, NHS dentistry: options for change was published. ${ }^{6}$ It proposed the initiation of demonstration projects to explore new models of working in the GDS. An application was submitted for such a site at Southend (now subsumed into South East Essex) PCT to organise and run a clinical audit pilot scheme alternative to the established GDS scheme. This ran from 2003 to 2005. The design of the Southend PCT scheme has been described elsewhere and included engaging GDPs in PCT-wide collaborative centrally-organised clinical audit. $^{7}$ The field site for the PCT scheme drew on previous work by the Southend PCT in other areas of NHS healthcare. ${ }^{5}$

Within each clinical audit, the design, analysis and report-writing were undertaken by the PCT; the clinicians' responsibilities were to collect the data and participate in the peer review of the results and in the required changes that were identified as a result of the audit. The analyses were carried out anonymously. The aggregated results were collated by the PCT and the individual results went directly to the clinician. All the dentists in contract with the PCT (not just those who participated in an audit) were sent the results and encouraged to attend a forum 
with their colleagues to learn from the outcomes. Between 2003 and 2005, three collaborative audits were run within the PCT area. In January 2010 an audit to assess the selection of recall intervals for patients according to their risk status was launched.

Routine six-monthly dental checkups have been customary for many patients in the general dental services in the United Kingdom for many years, however there appears very little evidence to support this as being an appropriate interval for all patients. ${ }^{8}$ In October 2004 the National Institute for Clinical Excellence (NICE) published guidelines recommending that the interval between reviews of patients' oral health be determined according to their clinical need. ${ }^{8}$ The guidance proposed that in order to be able to determine the regularity with which a patient is seen, a recall interval should be assigned for the patient that accounts for his/her specific need. They proposed that a risk assessment should be performed on each patient, taking account of their risk factors for oral disease. The patient could then be put into an appropriate risk category so that a recall interval could be assigned. ${ }^{8}$ Factors to be considered included the patient's susceptibility to developing dental caries, periodontal disease, oral cancer and their susceptibility to NCTSL. ${ }^{8}$

When using this guidance, once all of the risk factors have been examined, a patient can be placed in a risk category. If the patient is deemed a 'high risk' for one or more of the diseases outlined above, it is appropriate for them to be seen more frequently than a patient who has very low risk of developing oral or dental disease. The stated purpose of the NICE guidelines is to help clinicians assign recall intervals between oral health reviews that are appropriate to the needs of individual patients. The recommendations apply to patients of all ages. ${ }^{8}$ The move away from a routine six-month interval as the standard recall interval for a review of dental patients has proved a difficult one. ${ }^{9}$

\section{METHOD}

\section{Audit criteria}

The criteria selected for scrutiny in this audit project were:

- The categorisation of patients according to risk, by consideration of caries, periodontal disease, oral

\begin{tabular}{|l|l|l|}
\hline Audit Record No: & Yes & No \\
\hline (Please tick as appropriate) & & \\
\hline $\begin{array}{l}\text { 1. Has this patient been selected randomly for the sample? } \\
\text { (patients seem in the dental practice between 1st January to 31st December 2009) }\end{array}$ & & \\
\hline 2. Was a risk assessment done for caries and documented in the notes? & & \\
\hline 3. Was a risk assessment done for periodontal disease and documented in the notes? & & \\
\hline 4. Was a risk assessment done for oral cancer and documented in the notes? & $\begin{array}{l}\text { 5. Was a risk assessment done for non-carious tooth surface loss and } \\
\text { documented in the notes? }\end{array}$ & $\begin{array}{l}\text { 6. Whether an appropriate recall interval was selected and recorded in the notes? } \\
\text { (only record 'Yes' if associated with document risk assessment) }\end{array}$ \\
\hline $\begin{array}{l}\text { 7. Whether the discussion between the patient and the dentist about their recall } \\
\text { has been documented in the notes? }\end{array}$ & \\
\hline
\end{tabular}

Fig. 1 Retrospective data capture form

\begin{tabular}{|c|c|c|}
\hline Audit Record No: & Yes & No \\
\hline \multicolumn{3}{|l|}{ (Please tick as appropriate) } \\
\hline \multicolumn{3}{|l|}{$\begin{array}{l}\text { 1. Has this patient been selected randomly for the sample? } \\
\text { (patients seen in the dental practice in the month of February 2010) }\end{array}$} \\
\hline \multicolumn{3}{|l|}{ 2. Was a risk assessment done for caries and documented in the notes? } \\
\hline \multicolumn{3}{|l|}{ 3. Was a risk assessment done for periodontal disease and documented in the notes? } \\
\hline \multicolumn{3}{|l|}{ 4. Was a risk assessment done for oral cancer and documented in the notes? } \\
\hline \multicolumn{3}{|l|}{$\begin{array}{l}\text { 5. Was a risk assessment done for non-carious tooth surface loss and } \\
\text { documented in the notes? }\end{array}$} \\
\hline \multicolumn{3}{|l|}{ 6. Was the overall risk category assigned to the patient condition (low/medium/high)? } \\
\hline \multicolumn{3}{|l|}{ 7. Whether the level of risk category was documented in the notes? } \\
\hline \multicolumn{3}{|l|}{ 8. Whether an appropriate recall interval was selected and recorded in the notes? } \\
\hline \multicolumn{3}{|l|}{$\begin{array}{l}\text { 9. Whether the discussion between the patient and the dentist about their recall has } \\
\text { been documented in the notes? }\end{array}$} \\
\hline \multicolumn{3}{|l|}{ 10. Does the patient agree with the recommended recall interval? } \\
\hline \multicolumn{3}{|l|}{ 11. If no, please record the reasons for disagreement in the notes: } \\
\hline Any other comments: & & \\
\hline
\end{tabular}

\section{Fig. 2 Prospective data capture form}

cancer and NCTSL risk status and the documentation of the risk category in the notes

- The selection of an appropriate recall interval informed by the assessment of risk status for patients in general dental practices and the documentation of this in the notes

- The discussion of the recall interval selected with the patient and documentation of this in the notes.
These criteria were chosen following consideration of the NICE suggested audit criteria for carrying out an audit on the topic of recall intervals for patients in general dental practice. ${ }^{8}$

\section{Standard}

The audit followed the cycle of setting a standard, collecting data and then measuring the data against the standard. One previous clinical audit looking at the issue 
of recall intervals for patients in a general dental practice is present in the literature. ${ }^{10}$ This study used the standard that $80 \%$ of patients should be categorised according to their risk, have a recall interval appropriate for and informed by their risk status and have a discussion about their recall interval documented in their notes. The standard was adopted for this project.

\section{Design}

An introductory meeting was held with representatives from general dental practices across South East Essex PCT. The topic for audit was introduced and discussed, a variety of resources relating to assigning oral health risk status to individuals' oral health was provided, ${ }^{8,11}$ and some group work was undertaken by delegates in which they were asked to consider scenarios of fictitious patients in relation to various risk factors associated with caries, periodontal health, oral cancer and NCTSL, and assign a level of overall risk category for the patient.

Retrospective and prospective elements were included in the design of the audit project. To undertake the retrospective element, all GDPs were encouraged to collect data from 25 randomly selected record cards using a retrospective data capture form (Fig. 1) to check patients' records between 1 January 2009 and 31 December 2009 for evidence of presence or absence of the audit criteria.

To complete the prospective element of the audit, for the duration of February 2010, GDPs were asked that while they undertook examinations on NHS patients they should consider the patient's risk status in four areas (caries, periodontal disease, oral cancer and NCTSL), assign an overall risk category and an appropriate recall interval, discuss this with the patient and record these details in the patient's records. At the end of the month, the participants were asked to assess 25 randomly selected cards from examination appointments during February 2010 for evidence of presence or absence of the audit criteria. A prospective data capture form was provided for this purpose (Fig. 2). Once this data had been collected it was forwarded to South East Essex PCT where it was anonymised and then analysed using Microsoft Excel software. In addition, qualitative data was also collected from participants in the audit,

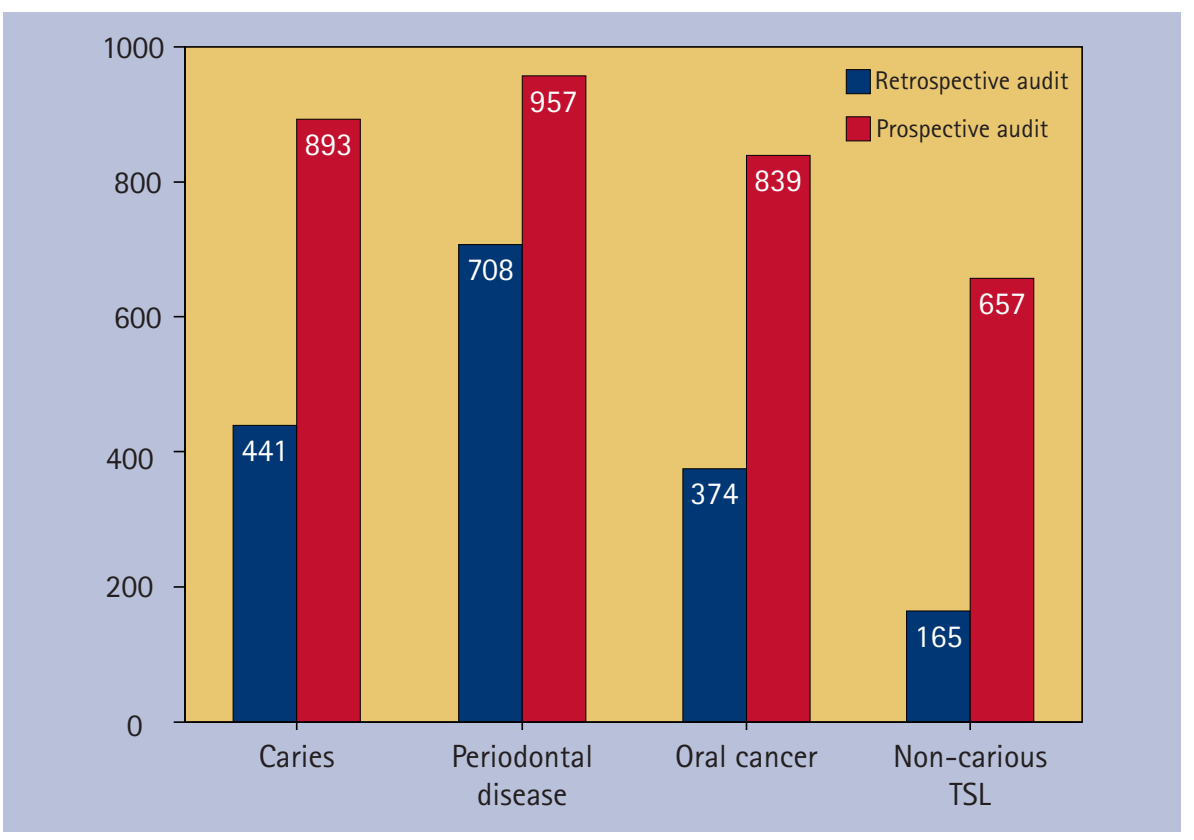

Fig. 3 Patients categorised according to risk; retrospective and prospective audit data

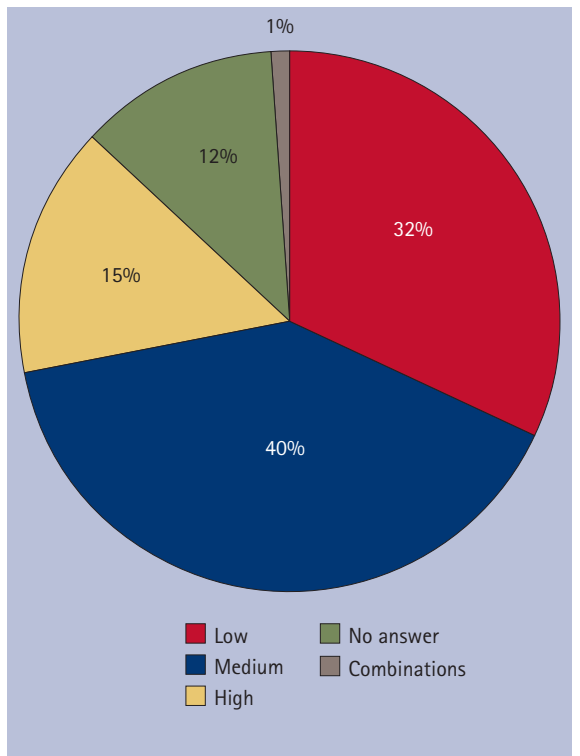

Fig. 4 The level of overall risk category assigned to each patient in prospective audit

who were encouraged to make comments regarding any aspect of the audit project when they submitted their data for analysis and at a post audit peer review meeting.

\section{RESULTS}

The total number of GDPs who participated in the retrospective audit was 39 , and in the prospective audit 42 .

\section{Categorisation of patients according to risk}

The results for categorisation of patients according to risk status for caries, periodontal disease, oral cancer and NCTSL for both retrospective and prospective

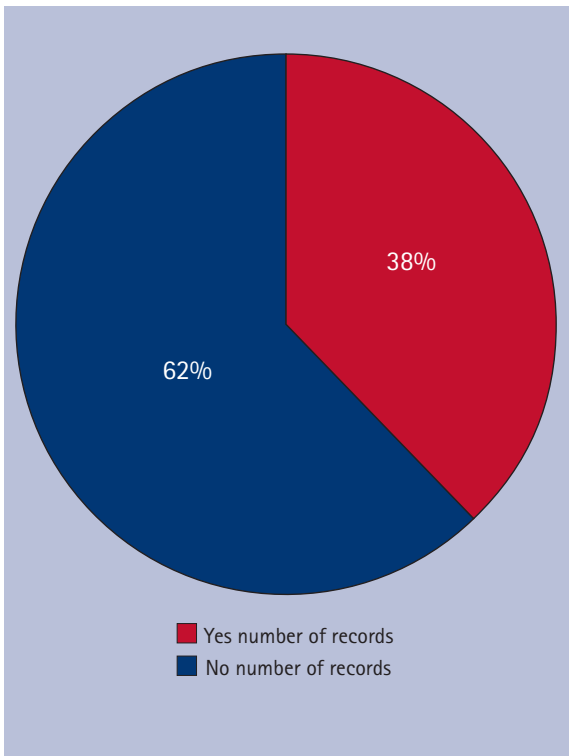

Fig. 5 Recall interval selected and recorded in notes, retrospective audit

elements of the audit are displayed in Figure 3. The retrospective data showed that most risk assessments were carried out for periodontal disease (70.8\%) and fewest risk assessments for NCTSL (16.5\%). The prospective data showed a higher number of risk assessments being carried out in all four areas than in the retrospective audit. As in the retrospective audit the smallest number of risk assessments were carried out for non-carious tooth surface loss (61.1\%). The results from the prospective audit showed that $87 \%$ of patients were assigned an overall risk category of low, medium or high risk (Fig. 4), exceeding the standard of $80 \%$ set for this criterion. 


\section{Appropriate recall interval selected and recorded in the patients' records}

The results from the retrospective audit indicated that an appropriate recall interval based on assessment of the patient's risk status was selected in 38\% of cases analysed (Fig. 5). The data from the prospective audit showed an increase to a level of $85 \%$, again exceeding the standard set of $80 \%$.

\section{Discussion occurred between patient and dentist regarding recall interval and was recorded in the patients' records}

The data from the retrospective audit indicated that a documented discussion between the dentist and the patient was occurring in only 11\% of cases reviewed. In the prospective audit this figure had risen to 65\%. This figure fell short of the standard set of $80 \%$, but nevertheless represented a marked increase on the figure from the retrospective data.

In addition to the results from quantitative data collected during the audit process, qualitative data collected from participants was grouped into the following themes.

\section{Reasons encountered for patients' resistance to recommended recall interval}

Some patients used to attending in family groups for their examination appointments felt that they would rather all attend together as a group for their subsequent examinations rather than returning at different intervals. On occasions when a patient was placed in a high-risk category and a shorter three-monthly recall interval was recommended, this was rejected by patients on the grounds of extra financial cost to the patient.

Conversely some patients when recommended a longer recall interval than they were used to, for instance one year rather than six months, still requested to be seen at a six-month interval for 'peace of mind'. Some patients felt that if too long an interval was recommended they may be more likely to forget to attend.

Other patients rejected the notion of the clinician determining an interval altogether, insisting that they wanted to be able to come in as and when they needed to.

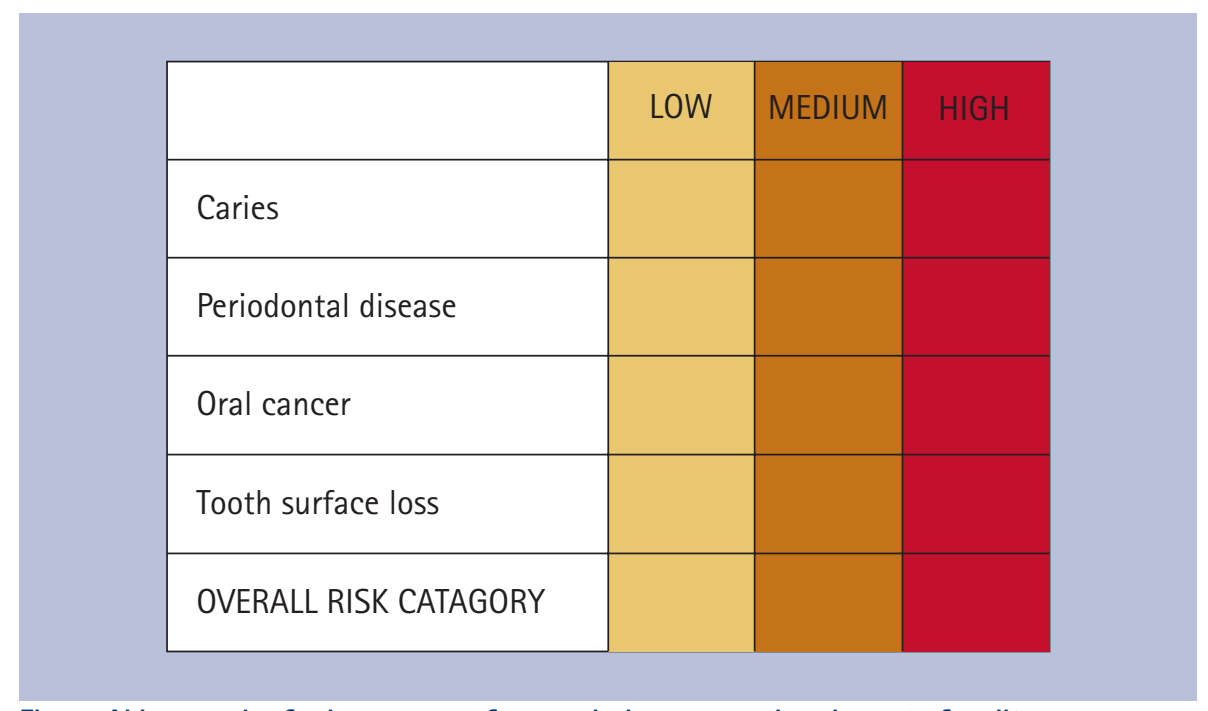

Fig. 6 Aide memoire for in-surgery reference during prospective element of audit

\section{Problems encountered with the suggested recall process}

Some participants felt that for children, recall intervals should also reflect the need for monitoring of orthodontic and occlusal development. Several participants found the extra tasks associated with discussion of risk and recall intervals with patients time consuming, although many also felt that they were having more discussion with patients about their oral health as a result. Many participants also felt that the whole concept of changing attitudes to recall intervals needs to be more aggressively promoted to patients, and that this should not be the sole responsibility of dental practices and their staff, but should be assisted by more input from the NHS and PCTs to inform the population at large and to help promote change. Several practices that participated in the audit involved many different members of the dental team; participants expressed the view that for a change to recall intervals to work well in a practice, a team approach is essential, with a champion from within the team supporting and inspiring the change in practice.

\section{Benefits associated with participation} in the recall audit

Many participants felt that taking part in the audit had caused them to think differently about how they went about undertaking an examination and what information they recorded and that this was a good thing both for the patient, in terms of more focused assessment of their oral health risk status and advice, and for the clinician as they may better record the activities they undertake in an examination. Several participants felt that the changes to their practising behaviour had persisted after the audit proper had been completed. An aide memoire (Fig. 6) designed to be used within the surgery was also considered by many participants to have been useful to 'jog the memory' and help make changes to practising behaviour.

\section{DISCUSSION}

The results from the retrospective audit risk assessments showed that only for periodontal disease were a majority of risk assessments carried out (70.8\%). For caries, oral cancer and NCTSL risk assessments were absent more than present and for NCTSL an assessment was recorded in only $16.5 \%$ of cases. One reason for the more widespread use of periodontal risk assessments may be the relatively high importance now placed on it by clinicians, through fear of litigation or complaints. Dental Protection report experiencing an increase in the level of complaints and litigation in dentistry, particularly in relation to periodontal disease. ${ }^{12}$

An assessment of NCTSL through erosion, abrasion, attrition and abfraction has been noted as a contributing factor when making an assessment of the oral health of an individual. ${ }^{8}$ Many clinicians may not think of NCTSL as an oral disease in the same way that caries, periodontal disease or oral cancer are. The lack of risk assessments in NCTSL in the retrospective audit (16.5\%), and also the lowest level risk assessment in the prospective audit (61.1\%) 
may indicate a current lack of knowledge and/or understanding of the importance of this area to an overall assessment of oral health.

The assignment of an overall risk category in the prospective audit was recorded at $87 \%$. Participants had been asked to score patients as overall low medium or high risk. The largest proportion of patient risk assessments recorded were medium, followed by low and then high risk.

The results for the selection of appropriate recall interval and recording of this in the patients' records showed a rise from $38 \%$ in the retrospective audit to $85 \%$ in the prospective audit. A greater rise was recorded for the criteria of 'discussion between the dentist and patient about their recall interval and recording of this in the patients' records', from 11\% to $65 \%$. Nevertheless, this was the lowest criterion recorded in both the retrospective and prospective elements to the audit. This is interesting in that it may indicate a tension between on the one hand providing effective communication with patients regarding their dental health and on the other the time constraints experienced by and expressed by some of the clinicians who participated in this audit. In particular there was a feeling that the onus of explaining the concept behind the recall interval linked to oral health risk lay with the dental practices and that this was timeconsuming and more could be done by bodies such as the Department of Health and PCTs to raise the public's awareness of these changes.

Several clinicians felt that the orthodontic assessment and monitoring of the developing dentition in children was an element that should be incorporated into an oral health risk assessment for children aimed at determining an appropriate recall interval. This is not described as a modifying factor to be considered by clinicians in the 2004 NICE guidelines on dental recall. ${ }^{8}$

Overall, all the results showed a marked improvement in the prospective audit in comparison with the retrospective audit. There were several aspects associated with the design of this audit that may have contributed to these improvements:
- The pre-audit introductory meeting had several elements to it. The background to the topic area of risk assessment and dental recall was presented

- A practitioner who had been implementing the NICE guidance in his practice for some time presented his experiences of doing this

- All the prospective participants in the audit took part in some group work looking at scenarios of fictitious patients to consider how they would determine a risk assessment for them and appropriate recall interval. The scenarios used were chosen from those detailed in the NICE guidance $2004^{8}$

- The process of how to complete the audit was then explained.

Participants were not required to undertake any of the design, data analysis or report writing elements of the audit, but were required to undertake collection of the data and be involved in the interpretation of the findings. Participants were also encouraged to look into the data to see how their individual results compared to those of their peers. In addition to the postaudit peer review meeting, the report was also sent to all practices in the PCT patch including those who did not participate in the audit project, in order to raise awareness of the audit undertaken, its subject matter and its findings and to encourage maximising engagement with as wide an audience as possible. Participants in the scheme felt that the audit should be repeated in 6 months to establish if the changes made to practising behaviour had persisted. The features used in the design and delivery of this audit project followed many of the recommendations made following a recent evaluation of clinical audit in dentistry. ${ }^{13}$

This audit project was undertaken in one particular PCT in Essex, involving 42 dentists. The generalisability of the results obtained is limited by the number of participants in the project and the geographic location in which it was undertaken.

\section{CONCLUSION}

The results from the retrospective element of this study indicated only limited use of a mechanism to select appropriate recall intervals for patients by reference to their risk of developing dental disease. Following brief educational input in the subject area of recall intervals a prospective audit showed a marked uptake by participants in categorising patients according to risk of dental disease, selecting recall intervals by reference to patients' risk status and in discussing the issue of recall interval with patients. This audit appears to have been successful as a tool to bring about change in the behaviour of dentists regarding their determination of appropriate recall intervals for patients. Nevertheless it remains to be seen how much, if any, of this behaviour change remains over time. A future re-audit undertaken once six months have elapsed from the completion of the original project will assess any lasting change.

The author would like to thank Sushmita Sinha, Matt Rangue and Gerard Cronin, from NHS South East Essex, and Dr Peter Martin and Professor Kimmy Eldridge from University of Essex for their support and assistance during this audit project.

1. Department of Health. Clinical audit in general dental practice. London: Department of Health, 1995.

2. Working group on audit in primary dental care. $A$ workbook. London: British Dental Association, 1993

3. Department of Health. Modernising NHS dentistry - clinical audit and peer review in the GDS. London: Department of Health, 2001

4. Department of Health. Standard GDS contract. London: Department of Health, 2005.

5. Cannell P J. Evaluation of the end user (dentist) experience of undertaking clinical audit in a PCT-led NHS modernisation agency pilot scheme. Prim Dent Care 2009; 16: 168-176.

6. Department of Health. NHS dentistry: options for change. London: The Stationery Office, 2002.

7. Cannell P J. Linking clinical audit in general dental services to primary care trust clinical governance progress report of an approach used in Southend. Prim Dent Care 2005; 12: 23-26.

8. National Institute of Clinical Excellence. Clinical Guideline 19. Dental recall: recall interval between routine dental examinations. London: National Institute of Clinical Excellence, 2004.

9. Department of Health. NHS dental services in England: an independent review by Professor Jimmy Steele. London: Department of Health, 2009.

10. Gibson C, Moosajee A. Selecting appropriate recall intervals for patients in general dental practice - an audit project to categorize patients according to risk. Dent Update 2008; 35: 188-194.

11. National Institute of Clinical Excellence. Quick reference guide: dental recall. London: National Institute of Clinical Excellence, 2004.

12. Dental Protection Limited. In safe hands: annual review 2010. London: Dental Protection Limited, 2010.

13 Cannell P J. Evaluation and comparison of the end user (dentist) experience of two approaches to undertaking clinical audit in the general dental services (GDS). The post April 2001 GDS scheme and a PCT led NHS Modernisation Agency pilot scheme. MSc Dissertation. Essex: University of Essex, 2009 